research support from: Genzyme Sanofi, GlaxoSmithKline, David Isenberg: None declared, Sarah Skeoch: None declared, Edward Vital Consultant of: Roche, GSK and AstraZeneca, Grant/research support from: GSK and AstraZeneca

DOI: 10.1136/annrheumdis-2021-eular.2496

\section{POS0749 ASSOCIATION BETWEEN IMMUNE-SEROLOGICAL PROFILE AND PULMONARY MANIFESTATIONS IN COLOMBIAN PATIENTS WITH SYSTEMIC LUPUS ERYTHEMATOSUS (LES)}

S. Herrera ${ }^{1}$, J. C. Diaz-Coronado ${ }^{1,2}$, S. Monsalve ${ }^{2}$, S. Guerra-Zarama ${ }^{2}$, M. F. Saavedra Chacón ${ }^{2}$, J. Barbosa ${ }^{2}$, J. D. Serna Giraldo², J. D. Lopez ${ }^{2}$, J. M. Gutiérrez ${ }^{2}$, T. Vega ${ }^{2}$, D. Orozco ${ }^{2}$, D. Ocampo ${ }^{2}$, N. Zuluaga ${ }^{2}$, D. HernandezParra ${ }^{1}$, D. Rojas-Gualdrón ${ }^{3}$, R. Pineda.Tamayo ${ }^{1} .{ }^{1}$ Artmedica, Clinical Information Group, Medell in, Colombia; ${ }^{2}$ CES University, Department of Internal Medicine, Medellín, Colombia; ${ }^{3}$ CES University, Facultad de Medicina, Medellín, Colombia

Background: Pulmonary involvement is common in Systemic Lupus Erythematosus (SLE) patients with varying degrees of parenchymal, vascular, and pleural compromise. In GLADEL, pulmonary involvement was reported in $28.4 \%$ of the cohort, but its occurrence ranges between $30-90 \%$ due to diversity in populations and the methods used to define it.

Objectives: To describe the immune-serological profile of a Colombian cohort of SLE patients and to establish its association with pulmonary manifestations.

Methods: Retrospective analysis of observational data from the follow-up of a cohort of adult patients with SLE. We included 559 patients that fulfilled the SLICC 2012 classification criteria with at least 6 months of disease history and being treated in a rheumatology specialized medical center between 2015 and 2018. The immuno-serological profile was characterized, and pulmonary involvement was monitored for 1 year. Diagnosis of pulmonary involvement was performed with the rheumatologist report in the clinical chart. Prevalence of pulmonary manifestations and immune-serological profile was determined, and logistic regression was performed afterward adjusted by age, sex, and level of education to establish the association between pulmonary manifestations and a positive auto-antibodies profile.

Results: The median age of the cohort was 45 years, $96.5 \%$ were female. Pulmonary involvement was documented in 113 patients $(20.5 \%)$ at the beginning of the study. Their frequency was: pleuritis $(14.3 \%)$, lupus pneumonitis $(3.6 \%)$, pulmonary hypertension (3.2\%), interstitial lung disease (ILD) $(2.3 \%)$, pulmonary embolism (2.3\%), lung fibrosis $(2.14 \%)$, alveolar hemorrhage (1.4\%), shrinking lung $(0.2 \%)$. At 1 year of follow up. there were no statistically significant differences in the frequency of pulmonary manifestations. As for the immune-serological profile, there were positive ANA in 92\%, anti-dsDNA in 53.1\%, anti-B2GP $\operatorname{lgM} 15.2 \%$, anti- B2GP IgG in $17.2 \%$, and ENA in $97.2 \%$; as for the ENA $41.7 \%$ had positive anti-RNP, $40.2 \%$ anti-Ro, $36.4 \%$ anti-SM and $16.5 \%$ anti-La. Low complement levels was characterized as follows: C3 $53.1 \%$ and C4 29.2\%. In the logistic regression adjusted by age, sex and level of education, there was an association between anti-SM and pulmonary manifestations with an adjusted OR of $1.85 ; 95 \% \mathrm{Cl} 1.13-3.01$

Conclusion: An association between anti-SM positivity and pleuro-pulmonary manifestations was found. In other cohorts with a greater size, anti-La and antiRNP have been associated with pulmonary involvement (OR $2.51 ; 95 \% \mathrm{Cl} 1.39$ 4.57 and OR 1.32; $95 \% \mathrm{Cl} 1-1.75$ respectively). Anti-RNP positivity has been associated in particular with ILD, pulmonary hypertension and shrinking lung. Although these manifestations prevalence was similar in our cohort, an association with this antibody was not found (OR 1.01, 95\% Cl: 0.2-4.9). This could be explained by the smaller sample size. As for anti-La positivity, its prevalence in our cohort was less than what was found in the GLADEL cohort (16.5\% vs $24.3 \%$ respectively). It is possible that this could explain the poor association between anti-La positivity with the presence of pulmonary manifestations in our study compared with those of the GLADEL cohort. There are data that indicates that anti-SM and anti-RNP simultaneous positivity is related mainly to pleuritis OR 1.98 (95\% Cl: 1.31-3); and this kind of involvement was found to be more frequent in our study. Our results suggest an association between positive anti-SM and pulmonary manifestations in Colombian patients with SLE, pleuritis in particular.

\section{REFERENCES:}

[1] Haye Salinas MJ, Caeiro F, Saurit V et al. Pleuropulmonary involvement in patients with systemic lupus erythematosus from a Latin American inception cohort (GLADEL). Lupus. 2017;26(13):1368-77.
[2] Emad Y, Gheita T, Darweesh H, Klooster P, et al. Antibodies to extractable nuclear antigens (ENAS) in systemic lupus erythematosus patients: Correlations with clinical manifestations and disease activity. Reumatismo. 2018;70(2):85-91.

Disclosure of Interests: None declared

DOI: 10.1136/annrheumdis-2021-eular.2577

\section{POS0750 THE CHARACTERISTICS OF SYSTEMIC LUPUS ERYTHEMATOSUS WHO HAVE CONSTITUTIONAL SYMPTOMS AND LYMPHADENOPATHY}

Y. Erez ${ }^{1}$, S. Gulle ${ }^{1}$, A. Karakas ${ }^{1}$, R. Bekis ${ }^{2}$, A. Balcl ${ }^{3}$, M. Birlik ${ }^{1}$, G. Can ${ }^{1}$, F. Onen ${ }^{1}$, I. Sari'. ${ }^{1}$ Dokuz Eylul University School of Medicine, Division of Rheumatology, Izzmir, Turkey; ${ }^{2}$ Dokuz Eylul University School of Medicine, Division of Nuclear Medicine, İzmir, Turkey; ${ }^{3}$ Dokuz Eylul University School of Medicine, Division of Radiology, İzmir, Turkey

Background: Systemic lupus erythematosus (SLE) is the prototype of systemic autoimmune diseases. It may affect any organ system and may present with a variety of clinical symptoms. Lymphadenopathy (LAP) is a nonspecific finding of SLE and often associated to the active disease state. LAP associated SLE pose a significant diagnostic challenge especially in the presence of constitutional symptoms.

Objectives: In this retrospective cohort study, we evaluated the clinical and imaging characteristics of SLE patients who presented with LAP and constitutional symptoms.

Methods: SLE Patients with lymphadenopathy who had constitutional symptoms were reviewed retrospectively. The characteristics of the patients such as age, sex, disease duration, clinical features, and laboratory tests including autoantibodies against ANA, dsDNA and ENA were recorded. Imaging findings such as positron emission tomography-computed tomography (PET/CT), thoracal or abdominal CT or diagnostic lymph node biopsy were identified.

Results: There were 28 patients in this study. The mean age at the time of LAP was $38.9 \pm 15.2$ years, $22(78.6 \%)$ were female. All patients were fulfilling SLICC 2012 classification criteria for SLE. LAP was the presenting feature of SLE in $15(53.6 \%)$ patients. The most common clinical features associated with LAP were arthritis $(42.8 \%)$, fever $(35.7 \%)$, acute lupus rash $(32.1 \%)$ and serositis $(28.5 \%)$. There were $11(39.2 \%)$ who had significant proteinuria and $4(14.3 \%)$ had class III or IV renal histology. Anti-phospholipid antibodies were present in nearly one-third of the patients and 12 of them had venous or arterial thrombosis or pregnancy co-morbidity. Cervical LAP was present in $57.1 \%$, axillary LAP in $82.1 \%$ and inguinal in $36 \%$. On CT, the prevalence of thoracal and abdominal LAP and hepatomegaly and splenomegaly were $61.5 \%, 37,5 \%, 40 \%$ and $16 \%$ respectively. The pericardial and pleural effusion and intra-abdominal free fluid were $19.2 \%, 30.8 \%$ and $10.2 \%$ respectively. There were $19(67.8 \%)$ patients who underwent PET/CT imaging and all were reported as reactive LAP. In terms of SUV max 7 (36.8\%) of them had a threshold $\geq 2.5 .17(60.7 \%)$ patients required lymph node biopsy; 2 did not include lymphoid tissue, $4(30.7 \%)$ with atypical lymphoid hyperplasia suggestive of viral infection and remaining were reactive lymphoid hyperplasia. Autoantibody testing showed that anti-dsDNA was positive in $66.7 \%$ and the most common anti-ENAs were anti-nucleosome (35.7\%) and anti-Sm-RNP (35.7\%) followed by anti-SSA (25\%), anti-ribosomal $\mathrm{P}(17.9 \%)$ and anti-histone (14\%). The clinical and laboratory features of the clinical and laboratory features of the group is summarized in Table 1.

Table 1. Data at the time of LAP

Age, years

Disease duration, months

SLE-DAI, median (min-max)

Sex, female $n(\%)$

Clinical features

Acute cutaneous

Arthritis

Fever

Other constitutional symptoms

Serositis

Renal involvement

Neurologic involvement

Leucopenia

Trombositopenia

Hemolitic anemia

ANA $(+)>1 / 320$

Anti-dsDNA $(+)$

Anti-Sm(+)

Anti-phospholipid antibody $(+)$

Low complement
$38.9 \pm 15.2$ $3(2-300)$ $9(2-30)$ $22(78.6)$ n (\%) 9 (32.1) 12 (42.8) $10(35.7)$ $18(64.2)$ $8(28.5)$ $11(39.2)$ 1 (3.6) 7 (25.0) $5(17.8)$ $7(25.0)$ $28(100)$ $18(66.7)$ $5(17.9)$ 